\title{
Robotic Transaxillary Parathyroid Surgery
}

\section{Muhammet Recai Mazlumoglu}

Inis State Hospital, Otorhinolaryngology Clinic, Erzurum, 25600, Turkey

*Corresponding author: Muhammet Recai Mazlumoglu MR, Inis State Hospital, Otorhinolaryngology Clinic, Erzurum, 25600, Turkey, Tel: +905424355835; E-mail: dr.mazlumoglu@gmail.com

Received Date: October 25, 2017; Accepted Date: November 07, 2017; Published Date: November 17, 2017

Copyright: $\odot 2017$ Mazlumoglu MR. This is an open-access article distributed under the terms of the Creative Commons Attribution License, which permits unrestricted use, distribution, and reproduction in any medium, provided the original author and source are credited.

\begin{abstract}
The neck is anatomically complex, and incisions in this region commonly result in cosmetic complaints. Surgeons have worked to reduce the size of incisions used in the neck region through the use of ancillary instruments. One of these instruments, the Da Vinci Robotic System, is able to reach the neck using a wide variety of approaches. These approaches were first pioneered in thyroid and parathyroid surgeries. The most commonly used approach in robotic parathyroid surgery is transaxillary. We conducted a literature search to analyze the areas of use, advantages, and disadvantages of robotic transaxillary parathyroid surgery. The transaxillary approach does not create a scar in the neck region and significantly improves cosmetic outcomes for the patient, compared with conventional parathyroid surgery.
\end{abstract}

Keywords: Robot; Transaxillary; Surgery; Parathyroid

\section{Introduction}

Primary hyperparathyroidism (PHPT) is a common endocrine disease, and the treatment is primarily surgical. In the 1970s, parathyroid resection was done through a large incision made in the neck that allowed for full exploration and exposure of all four parathyroid glands. This method had a success rate of $97 \%$ there were complications in $1-2 \%$ of cases [1]. Typically, parathyroid adenomas are found only in a single gland, and targeted therapy can be performed that removes only the pathological gland. The 1980s saw the introduction of first one-sided two-gland, and then single-gland surgeries, made possible by the development of more advanced preoperative imaging methods and the introduction of intraoperative parathyroid hormone monitoring $[1,2]$.

Further refinements focused on reducing hospitalization time, postoperative pain, and improving cosmetic results. This resulted in the development of minimally invasive endoscopic and videoscopic surgical techniques. However, there were drawbacks to these methods, including a reduced field of view, difficulty manipulating instruments, and the insuflation of $\mathrm{CO}_{2}$ into the neck tissues. Robotic transaxillary parathyroid surgery (RTAPS), using the da Vinci Surgical System (Intuitive Surgical, Sunnyvale, CA, USA) aims to address some of the issues with current minimally invasive techniques $[3,4]$. There have been several studies reporting on the use of RTAPS published in recent years. Landry and Tolley published the first case series on this subject in 2011.

\section{Preoperative Evaluation of Parathyroid Pathology}

The type and location of parathyroid pathology can be determined using several imaging modalities including high-resolution ultrasound (USG), Tc-99m sestamibi scintigraphy (MIBI), computed tomography (CT), magnetic resonance imaging (MRI), and positron emission tomography (PET). Of these, USG, Tc-99m scintigraphy, and CT are the most commonly used methods [5]. On USG, a parathyroid adenoma typically appears hypoechoic, oval-shaped, and homogenous.
In patients with posterior or ectopic lesions or thick necks, MIBI scintigraphy can be very useful in localizing pathology. CT is useful for identifying masses resident in atypical sites, including ectopic lesions and lesions present in the tracheoesophageal grooves. Fourdimensional computed tomography (4D-CT) is the most advanced of CT [6]. MIBI together with USG has a diagnostic sensitivity of $95 \%$ and is the most frequently used of the methods described above [5]. $\mathrm{CT}, \mathrm{PET}$, and selective venous sampling are rarely used in the reported literature.

\section{Intraoperative Assessment in Parathyroid Surgery}

Radio-Guided Parathyroidectomy (RGP) and intraoperative parathyroid hormone (IOPTH) assays are both methods for intraoperative assessment in parathyroid surgery. RGP is a type of MIBI scan, where the isotope is given 1-2 $\mathrm{h}$ before surgery, and a gamma probe is used to localize the adenoma intraoperatively. IOPTH is also commonly used for monitoring the successful resection of the pathological gland [6,7]. Following gland resection, the drop in parathyroid hormone is rapid, and occurs within 1.5-21.5 min. Surgery can be safely terminated once IOPTH measurement shows a $50 \%$ drop in parathyroid hormone from preoperative levels. In our review of the literature, IOPTH assays are much more widely used than RPG $[8,9]$.

\section{Patient Selection}

There is currently no consensus on the criteria for selecting patients for RTAPS. In general, the ideal patient has some or all of the following characteristics: sporadic PHPT; signal gland disease, as suggested in preoperative ultrasonography and/or MIBI scan; a body mass index $(\mathrm{BMI})<30$; hypertrophic scars or keloid formation (particularly in young patients); no goiter; no previous radiation or surgical intervention in the neck region; no parathyroid carcinoma; no suspected multinodular goiter; and no cervical spinal disease $[10,11]$. Although previous reports show RTAPS is generally applied in cases of single, sporadic parathyroid adenomas, there are several reports of surgical cases that do not conform to the above criteria. Garcia-Roca et 
al. [7] used RTAPS to retrieve a single parathyroid gland from a patient who was also donating their kidney for renal transplantation. Mohsin et al. [9] performed robotic-assisted parathyroid surgery in conjunction with thyroid lobectomy in a patient with suspected parathyroid carcinoma, despite the fact that parathyroid carcinoma can be considered a contraindication. Noureldine et al. [4] performed simultaneous thyroid lobectomy with parathyroidectomy in three patients in nine trials. Foley et al. [12] performed RTAPS in patients with a BMI $<29$, a criterion that was different from the rest of the general literature. Across all studies, RTAPS was commonly performed in patients who did not want a visible scar on the neck.

\section{Operation}

RTAPS is generally performed with three robotic instruments (a Maryland dissector, harmonic curved shears, and ProGrasp forceps), a 30-degree endoscope, and a dual-channel camera. Forceps, retractors, and electrocautery are required for dissection and elevation. Equipment for laryngeal nerve monitoring is also recommended [3].

The position of the patient is important for this approach. While under general anesthesia, the patient is positioned supine with the neck slightly extended. The arm ipsilateral to the lesion is placed cephalad and flexed, above the head. This position increases the exposure of the axilla, and reduces the distance between the axilla and the parathyroid glands [8]. After proper positioning, Noureldine et al. [4] reported taking blood to measured baseline PTH levels. In some reports, the exact location of the lesion was visualized with USG before the initial incision $[10,13]$.

An initial transverse incision is made from the lateral aspect of the sternal notch to the axilla. The lower limit is directed posteriorly to make the incision less visible. An oblique, 60-degree line is made from the thyrohyoid membrane to the axilla, and is combined with the line of the initial incision. Next, a 5-6 cm incision is made from where these two lines intersect. The subplatysmal flap is advanced through the pectoral fascia. A window is opened between the head of the sternum and the head of the clavicle. Next, the sternal head and strap muscles are elevated using a special retractor to reach the thyroid surface. The da Vinci Surgical System (Intuitive Surgical) is then docked and robotic arms are attached [9]. Finally, the thyroid are retracted inferiorly and medially, sternocleidomastoid muscle laterally. The recurrent laryngeal nerve can be monitored in the trachea-esophageal groove. Once parathyroid tissue is identified, it can be confirmed with a gamma probe. The parathyroid tissue is then removed. Successful removal is confirmed using an IOPTH assay. The operation finishes with the placement of a drain [9].

The postoperative follow-up procedure for RTAPS is the same as in open techniques. In some cases, patients are discharged the same day as surgery, with a cold-compress on the wound. In other cases, patients are admitted for observation for one or more days. On postoperative day two or three, the drain is removed and the wound is checked [3].

Common complications in open parathyroid surgery include hematoma, airway obstruction, recurrent laryngeal nerve injury, tracheal injury, vascular injury, and postoperative hypocalcemia [12-14]. These complications can also occur in RTAPS. Additional complications specific to RTAPS include brachial plexus stretch injury, loss of sensation in the periaxillary skin, and weakness and pain in the shoulder.

The RTAPS technique has several disadvantages, including expense, long operating times, a requirement for experienced surgeons, and application in only select patients. However, RTAPS can safely be applied in patients that are concerned about the cosmetic appearance of conventional surgical techniques. Further research is required, particularly studies with large numbers of cases and prospective, randomized trials that compare RTAPS directly to conventional open techniques.

\section{References}

1. Brunaud L, Li Z, Van Den Heede K, Cuny T, Van Slycke S, et al. (2016) Endoscopic and robotic parathyroidectomy in patients with primary hyperparathyroidism. Gland Surg 5: 352-360.

2. James BC, Kaplan EL, Grogan RH, Angelos P (2015) What's in a name?: Providing clarity in the definition of minimally invasive parathyroidectomy. World J Surg 39: 975-980.

3. Mohamed HE, Bhatia P, Aslam R, Moulthrop T, Kandil E (2015) Robotic transaxillary and retroauricular parathyroid surgery. Gland Surgery 4: 420-428.

4. Noureldine SI, Lewing N, Tufano RP, Kandil E (2014) The Role of the Robotic-Assisted Transaxillary Gasless Approach for the Removal of Parathyroid Adenomas. ORL J Otorhinolaryngol Relat Spec 76: 19-24.

5. Lumachi F, Zucchetta P, Marzola MC, Boccagni P, Angelini F, et al. (2000) Advantages of combined technetium-99m sestamibi scintigraphy and high-resolution ultrasonography in parathyroid localization: comparative study in 91 patients with primary hyperparathyroidism. Eur J Endocrinol 143: 755-760.

6. Chazen JL, Gupta A, Dunning A, Phillips CD (2012) Diagnostic accuracy of $4 \mathrm{D}-\mathrm{CT}$ for parathyroid adenomas and hyperplasia. AJNR Am J Neuroradiol 33: 429-433.

7. Garcia-Roca R, Garcia-Aroz S, Tzvetanov IG, Giulianotti PC, Campara M, et al. (2016) Simultaneous Living Donor Kidney and Parathyroid Allotransplantation: First Case Report and Review of Literature. Transplatation 100: 1318-1321.

8. Li X, Massasati SA, Kandil E (2012) Single incision robotic transaxillary approach to perform parathyroidectomy. Gland Surg 1: 169-170.

9. Mohsin K, Alzahrani H, Ali DB, Kang SW, Kandil E (2017) Robotic transaxillary parathyroidectomy. Gland Surg 6: 410-411.

10. Katz L, Khalek MA, Crawford B, Kandil E (2012) Robotic-assisted transaxillary parathyroidectomy of an atypical adenoma. Minim Invasive Ther Allied Technol 21: 201-205.

11. Landry CS, Grubbs EG, Morris GS, Turner NS, Holsinger FC (2011) Robot assisted transaxillary surgery (RATS) for the removal of thyroid and parathyroid glands. Surgery 149: 549-555.

12. Foley CS, Agcaoglu O, Siperstein AE, Berber E (2012) Robotic transaxillary endocrine surgery: a comparison with conventional open technique. Surg Endosc 26: 2259-2266.

13. Karagkounis G, Uzun DD, Mason DP, Murthy SC, Berber E (2014) Robotic surgery for primary hyperparathyroidism. Surg Endosc 28: 2702-2707.

14. Tolley N, Arora A, Palazzo F, Garas G, Dhawan R (2011) Robotic-assisted parathyroidectomy: a feasibility study. Otolaryngol Head Neck Surg 144: 859-866. 